\title{
Poor adherence to antihypertensive drugs
}

In this 10-minute consultation by Mohammed Awais Hameed and colleagues (BMJ 2016;354:i3268, doi:10.1136/bmj.i3268), the final sentence of the first paragraph under the heading "Discuss risk" should not have read: "For example, 'In a population of 100 people aged 40-69, with every $2 \mathrm{~mm} \mathrm{Hg}$ rise in blood pressure, 10 people are at risk of dying from a stroke and seven people are at a risk of dying from heart disease such as a heart attack."
The increased risk of stroke and cardiac mortality with each 2 $\mathrm{mm} \mathrm{Hg}$ increase in systolic blood pressure in the second sentence of the paragraph refers to relative risk, and so this example is misleading.

The sentence should have read, "For example, if a middle aged person's current risk of death from heart attack or stroke is $20 \%$ in the next 10 years, this risk is likely to increase to $21.4 \%$ or $22 \%$ respectively if blood pressure rises by only $2 \mathrm{~mm} \mathrm{Hg}$ from the current reading." 